\title{
PReS-FINAL-2074: The role of the probiotic VSL-3 as adjuvant therapy in patients with undifferentiated spondyloarthritis (ERA)
}

\author{
M Vidovic ${ }^{*}$, M Perica, L Lamot, M Zaninovic, L Tambic Bukovac, M Harjacek \\ From 20th Pediatric Rheumatology European Society (PReS) Congress \\ Ljubljana, Slovenia. 25-29 September 2013
}

\begin{abstract}
Introduction
Calprotectin is a neutrophil derived protein that binds calcium and belongs to the $\mathrm{S} 100$ family. It can be quantified in feces and has become established as a marker of gut inflammation, where increased levels are a direct result of neutrophil migration into the gut lumen across the inflamed mucosa. Since subclinical gut inflammation is present in the majority of adult and pediatric spondyloarthritis patients, fecal calprotectin (fcal) is emerging as possible noninvasive biomarker. There is strong evidence supporting the role of the VSL-3 probiotic in decreasing fcal, thus promoting and maintaining remission in patients with inflammatory bowel disease (IBD), but very little is known of its potential effects on disease activity in children with undifferentiated spondyloarthritis (ERA).
\end{abstract}

\section{Objectives}

To assess the effect of the VSL-3 probiotic on fcal levels, clinical symptoms and disease activity of children with undifferentiated spondyloarthritis (ERA).

\section{Methods}

Sixteen patients diagnosed with ERA, according to the ILAR criteria, were treated with VSL-3, in addition to standard therapy (NSAID's and DMARD's). All patients were negative for gastrointestinal (GI) symptoms, and/or poor growth. All four ankylosing spondylitis patients received biologics; two were treated with adalimumab and further two with infliximab. In addition to general clinical data, patients completed the BASFI and BASDAI questioners, ESR and CRP were obtained, and fcal was measured by the Calprest ELISA method (Eurospital Spa, Italy). After VSL-3 was introduced, two follow up visits were scheduled; the

\footnotetext{
Childrens Hospital Srebrnjak, Zagreb, Croatia
} 